\title{
INTOLERANCE OF UNCERTAINTY, ANXIETY AND DEPRESSION IN THE CONTEXT OF COVID-19 IN INDONESIA
}

\begin{tabular}{l}
\hline \hline Muhamad Salis Yuniardi $^{1}$, Matthew Cant $^{* 2}$, Hanif Akhtar $^{3}$ \\
${ }^{1}$ Psychology Faculty, University of Muhammadiyah Malang, Malang, Indonesia. \\
${ }^{2}$ Psychology Faculty, University of Muhammadiyah Malang, Malang, Indonesia. \\
${ }^{3}$ Psychology Faculty, University of Muhammadiyah Malang, Malang, Indonesia. \\
\hline \hline
\end{tabular}

\begin{abstract}
This Covid-19 pandemic brings uncertainty and may lead to mental problems. Yet, in this context, no study has examined the relationship between intolerance of uncertainty (IU) and either anxiety or depression during pandemic among Indonesians. This study investigated the pathway between IU, anxiety and depression serially mediated by cognitive appraisals (perceived severity, controllability, and knowledge) and emotional and behavioural responses to COVID-19. Online psychological measures were administered to 406 participants, including the IUS-12, the DASS-21, cognitive appraisal scales, and emotional and behavioural response scale. An indirect pathway was found between IU and depression, and the effect was serially mediated by perceived of severity and emotional and behavioural responses to COVID-19. Perceived knowledge and perceived controllability of COVID-19 did not significantly contribute to the model. A similar pathway was found for IU predicting anxiety. As conclusion, IU represents a risk factor for heightened emotional and behavioural difficulties that eventually, could lead to either anxiety or depression during COVID-19.
\end{abstract}

Keywords: Indonesia; COVID-19; Intolerance of Uncertainty; Anxiety, Depression; Cognitive Appraisals; Emotional and Behavioural responses

\section{Introduction}

The first cases of SARS-CoV-2 coronavirus (COVID-19) were confirmed in Indonesia on the $2^{\text {nd }}$ of March, 2020. Cases have continued to rise across the country. Government responses to the pandemic have been mixed, with large scale

\footnotetext{
* Correspondence concerning this article should be addressed to Matthew Cant, Psychology Faculty, University of Muhammadiyah Malang, Malang, Indonesia.

Email: matthew_cant@aol.com
} 
social restrictions being enforced at different times and with varying degrees of rigour. The consequences of the virus itself, as well as the social, psychological and economic ramifications can be observed across the country (Suryahadi et al., 2020). This has been compounded by an inconsistent implementation of preventative measures and a lacking coherent scientific government message. Accordingly, many have sought refuge in unscientific and unsupported information on the virus (i.e., cures, transmission) (Koran Tempo, 2020), leading to a greater sense of uncertainty. It's important, therefore, to understand what factors underlie adverse psychological outcomes for different forms of mental-illness and the role of uncertainty.

Psychological distress seems to be accompanied by an increasing prevalence of disorders such as depression and anxiety. A number of Indonesian studies report a moderate-high prevalence (e.g. Almira, 2020; Mulya et al., 2021; Sunjaya et al., 2021). Although some level of depressive and anxious symptoms are to be expected due to the pandemic's nature, this is troubling, given that depression and anxiety are associated with functional impairments and increased likelihood of suicide (Parker, 2020; Pompili, 2019), which could have lasting effects after the pandemic has subsided.

A mental-health survey conducted online by the Association of Indonesian Mental Medicine Specialists (PDSKJI) found that out of 1,552 participants in the regions of West Java (23.4\%), DKI Jakarta (16.9\%), Central Java (15.5\%), and East Java (12.8\%); 66\% reported experiences of depression (i.e., sleep disturbance, decreased interest, fatigue, lack of energy, and decreased confidence) and $63 \%$ reported symptoms of anxiety (i.e., excessive worry about potential negative outcomes, difficulty relaxing and irritability). Another study using the Beck Depression Inventory-II questionnaire (BDI II) found that among college students, $21 \%$ of students experienced mild depression, $17 \%$ moderate depression and $34 \%$ severe depression (Hasanah et al., 2020).

Social restrictions and disruptions to daily life have led to an increased reliance on technology for tasks such as work, study and communication. Siste et al (2020) examined the effects of the pandemic on a number of variables, including internet addiction, psychopathological symptoms, and sleep quality. They found that internet addiction was significantly higher for participants living in a household with confirmed/suspected COVID-19 cases and it relates to higher psychopathological symptoms and low sleep quality. Importantly, the highest correlation with internet addiction and highest scores in general for participants with actual/potential COVID19 contact was depression. It's important to note that $66.8 \%$ of subjects reported residence in provinces that had not implemented the PSBB (social restrictions), highlighting the inconsistency between provinces and guidance on how to appropriately manage life during the pandemic.

Finally, this notion is supported by research from other countries. Global evidence indicates that there has been a spike in psychological distress and symptoms of mental illness (Bao et al., 2020), and some researchers have even 
reported possible collective trauma (Garfin, Silver, \& Holman, 2020). Wang et al (2020) recorded immediate psychological responses from 1210 participants from 194 cities in China, 3 weeks after the initial outbreak. Over half of participants (53.8\%) had rated the psychological impact as moderate or severe, while moderate to severe symptoms were reported by $16.5 \%$ participants for depression and $28.8 \%$ for anxiety. In Europe, González-Sanguino et al (2020), with a sample of 3,480 Spanish residents, found that $19 \%$ of participants met the threshold for depression, as did $22 \%$ for anxiety. Thus, factors that contribute to depression and its relationship with uncertainty need to be examined.

A characteristic of any novel virus outbreak is ambiguity and uncertainty, as the precise cause and severity is at first unknown, and the uncertainty in itself can increase psychosocial morbidity (Desclaux et al., 2017; DiGiovanni et al., 2004). Intolerance of Uncertainty (IU), (Freeston et al., 1994), therefore, the distress caused by not knowing - or having insufficient evidence to know - the outcome or experience of a given event, could heighten distress during COVID-19. IU can result in a range of cognitive, emotional and behavioural responses for the purpose of resolving or avoiding the distressing experience (Carleton, 2016).

Past research on IU and virus pandemics has mainly focused on anxiety. This is understandable as IU initially emerged from and displays strong empirical links with research on Generalized Anxiety Disorder (GAD) and related constructs (such as worry) (Freeston et al., 2020). For example, Taha et al (2014) found that IU increased participants perceptions of threat toward the H1N1 virus, which, alongside emotion-focused coping strategies, correlated with increased health anxiety. However IU later become known as a trans-diagnostic issue of psychological difficulties across a range of anxiety (Yuniardi, 2020) and non-anxiety disorders (Rosser, 2019), including depression. Pre-pandemic literature on the association between IU and depression demonstrates a strong relationship between the two even in the absence of anxiety (e.g. Carleton, 2012; de Jong-Meyer et al., 2009; Dugas et al., 2004; Gentes \& Ruscio, 2011; Mahoney \& McEvoy, 2012; McEvoy \& Mahoney, 2011; Miranda et al., 2008; Paulus et al., 2015; Yook et al., 2010).

Other researchers argue that this relationship can be fully accounted for by anxiety after methodological and conceptual issues have been properly examined (Boelen et al., 2010; Boelen \& Reijntjes, 2009; Khawaja \& McMahon, 2011). Jensen et al (2016), for example, found that while trait anxiety and depression correlated with IU (i.e., general factor, prospective IU and inhibitory IU) when entered into hierarchical analyses separately, only anxiety did when entered. The exception was that depression moderately but independently correlated with inhibitory IU in the undergraduate sample, even when entered simultaneously. This provides at least some evidence for an independent link between IU and depression.

Liao and Wei (2011) provided evidence for the mediating and moderating effects of rumination between IU, depression and anxiety. They found there was a significant rumination effect in the association between IU and depressive symptoms, which was enhanced by high levels of rumination. Furthermore, 
rumination fully mediated the association between IU and depression and only partially for IU and anxiety symptoms. Thus, empirical and conceptual links between IU and depression exist. While rumination is only one example, there may be more indirect and direct links between IU and depression, such as passive coping styles and the paralyzing nature of IU. Importantly, while there are many studies linking IU to anxiety during pandemics, to the authors' awareness, this relationship has not been examined for depression.

One clear similarity between IU, anxiety and depression is the dysfunction at cognitive, emotional and behavioural levels of functioning. IU has been known to cause alterations in certain cognitive processes relating to uncertainty (demonstrated through correlational and experimental studies). Focusing on uncertain aspects of an event has been characterized as uncertainty based reasoning (Reuman et al., 2015), and tendencies to overestimate the likelihood of negative events and IU has been well documented (e.g. Dugas et al., 2004, 2005; Koerner \& Dugas, 2008; Ladouceur, 2004; Ladouceur et al., 1997), with uncertainty being estimated as threatening. There is also substantial evidence that IU associates with a series of alterations in different neural and physiological networks associated with threat, such as increased reactivity to uncertainty represented by heightened activity of the amygdala and anterior insula (for review see Tanovic et al., 2018).

Accordingly, research has demonstrated that IU can alter cognitive appraisals of virus pandemics. Cognitive appraisals are the interpretation of events that happen to an individual (Lazarus, 1966), and play a major role in determining the psychobiological responses and action tendencies to that event (Mehu \& Scherer, 2015). That is, cognitive appraisals are heavily involved in the bodies stress response to perceived threats, and afford an adaptive psychological mechanism, whereby, individuals can enhance a response to a situation by decoupling response and stimulus (Scherer, 1984).

However, maladaptive cognitive appraisals can increase psychological distress and vulnerability to emotional disorders, articulated by the framework of cognitive risk factors and emotional dysfunction (Cox et al., 2001; Roberts et al., 1998; Spasojević \& Alloy, 2001). During COVID-19, Li et al (2020) found that perceived severity was associated with adverse emotional and behavioural outcomes (i.e., negative emotion, positive emotion, sleep problems, aggression and so forth), consistent with previous studies (Prasetyo et al., 2020; Xin et al., 2020; Yıldırım \& Güler, 2020; Zhong et al., 2020).

Strong situational evaluations are associated with heightened emotional responses (Mehu \& Scherer, 2015), and the more intense the response, the more need for processes of emotional regulation. However, this can be disrupted by various deficits and bias's in cognition, which can impair an individual's ability to regulate emotions effectively, thereby increasing vulnerabilities to emotional disorders (Joormann \& Gotlib, 2010). Many studies demonstrate the link between cognitive 
appraisals and depression, such as in breast cancer patients (Bigatti et al., 2012), and long-term depressive symptoms have been predicted by the link between daily cognitive appraisals and negative affect (Lee-Flynn et al., 2011). Taha et al (2014) demonstrates how IU can serve to increase anxiety during virus pandemics through the alterations of cognitive appraisals and subsequent reliance on maladaptive emotion-focused coping strategies. It's likely that a similar pathway is operating during the current pandemic. However, should IU represent a transdiagnostic construct of psychological difficulties, and cause the perception of uncertainty itself to appear threatening and lead to adverse emotional and behavioural outcomes; it's important to examine these associations for depression during COVID-19. Given the high levels of uncertainty surround the current pandemic for Indonesian's, this is required to understand what role IU is playing in both anxiety and depression.

The main goal of this study, then, is to investigate indirect links between IU and depression for Indonesian's during COVID-19. Of particular interest is the role of IU in disrupting cognitive, emotional and behavioural responses to the pandemic. However, this study also aims to provide further evidence for the role of IU for increasing anxiety. Thus, two hypothesized models are proposed. First, that IU forms an indirect pathway with and thereby increases depression. This pathway will be serially mediated by three cognitive appraisal styles - perceived knowledge, perceived severity, and perceived controllability of COVID-19 - and emotional and behavioural responses (i.e., increased negative affect, lower positive affect, anxious symptoms, and sleep disturbance). Secondly, that IU increases anxiety via the same route in a separate model.

\section{Method}

\section{Participants}

Participants were recruited through an online questionnaire developed on Google Forms. We collected valid data from 406 Indonesians from various regions, all of which provided consent. The data analysis excluded cases with missing values.

There were 293 females (72\%) and 113 males (28\%). The mean of the age was $27.69(\mathrm{SD}=9.96) ; 250$ participants $(61.58 \%)$ were between $15-24$ years of age, 54 participants (13.30\%) were between $25-34$ years of age, 75 participants $(18.47 \%)$ were between $35-44$ years of age, 18 participants $(4.43 \%)$ were between $45-55$ years of age, and 9 participants $(2.22 \%)$ were above 55 years of age. More than half of participants were college students $(58.62 \%)$. The complete demographic characteristics of the participants can be seen in Table 1. 
Table 1. Demographic characteristics of participants

\begin{tabular}{ccc}
\hline Variables & Frequency & $\mathbf{\%}$ \\
\hline Gender & & \\
Male & 113 & 27.83 \\
Female & 293 & 72.17 \\
\hline \multicolumn{3}{c}{ Age } \\
$18-24$ & 250 & 61.58 \\
$25-34$ & 54 & 13.3 \\
$35-44$ & 75 & 18.47 \\
$45-55$ & 18 & 4.43 \\
$>55$ & 9 & 2.22 \\
\hline
\end{tabular}

\begin{tabular}{ccc}
\hline & Education & \\
High school & 15 & 3.69 \\
Bachelor & 281 & 69.21 \\
Master & 92 & 22.66 \\
Doctor & 18 & 4.43 \\
\hline & Job & \\
Full-time job & 110 & 27.09 \\
Part-time job & 33 & 8.13 \\
Student & 238 & 58.62 \\
Not-working & 22 & 5.42 \\
Pension & 3 & 0.74 \\
\hline Total & 406 & 100 \\
\hline
\end{tabular}

Notes: total population $=406$. Mean participant age was $27.69(\mathrm{SD}=9.96)$. All participants were Indonesian.

\section{Measures}

Intolerance of Uncertainty Short Form (IUS-12)

Participants' IU scores were recorded using the Indonesia version of IUS-12 (Yuniardi, 2020). IUS-12 was validated by Carleton, Norton and Asmundson (2007) based on the original IUS-27 (Freeston et al., 1994). This measure employs 12 items for examining ones tendency to find uncertain situations distressing (e.g. "When it's time to act, uncertainty paralyses me", "I always want to know what the future has in store for me") on a five-point Likert scale ( 1 = "Not at all characteristic of me", 5 = "Entirely characteristic of me"). The total score was used as it correlates sufficiently with different psychopathological concepts (e.g. Khawaja \& Yu, 2010). Higher scores indicated greater IU. The internal consistency of this scale was excellent in the current sample (Cronbach's alpha $=0.83$ ). 
Depression, Anxiety and Stress Scale (DASS-21)

The DASS-21 was designed to measure emotional distress in three sub categories (Lovibond \& Lovibond, 1995): depression (e.g. loss of selfesteem/incentives and depressed mood), anxiety (e.g. fear and anticipation of negative events) and stress (e.g. persistent state of over arousal and low frustration tolerance). The DASS-21 is a self-report questionnaire with 21 items (seven items for each category) based on a four-point Likert scale. Participants were asked to rate to what extent each statement applied to them over the past week, with " $0=\operatorname{did}$ not apply to me at all" to " $3=$ applied to me very much, or most of the time". To calculate comparable scores with full DASS, the 7-items of depression and anxiety were multiplied by two. The higher the score the more severe the emotional distress was. However, only score from depression and anxiety were involved in the analysis of this study. Items included, "I found it hard to wind down", "I was aware of dryness of my month" and "I couldn't seem to experience any positive feeling at all". The internal consistency of this scale was good (Cronbach's alpha $=0.83$ ).

Permission was granted from $\mathrm{Li}$ et al (2020) for the use of the following scales; developed for recording mental-health during COVID-19 in China. All scales demonstrated strong internal validity and psychometric properties.

\section{Emotional and Behavioural Responses}

Emotional and Behavioural responses were recorded using 20 items, which measured a number of dimensions including: Negative emotion ( 8 items, anxiety, worry, depressive, lonely, sadness, anger, nervous and panic), positive emotions (3 items, joy, happiness, excitedness) sleep difficulties (4 items, insomnia, light sleep, nightmares and lack of sleep), aggression ( 2 items, argumentative and physical aggression), use of substances ( 2 items, smoking and drinking), and mobile use (1 item). Participants scores were recorded using a five-point Likert scale, which asked them to compare these facets after the outbreak with ones before (from " $1=$ much less compared to the days before the outbreak" to " $5=$ much more compared to the days before the outbreak"). Positive emotion was reverse scored to comply with negative dimensions. Lastly, a higher score indicated more negative emotion, less positive emotion, sleep difficulties, aggression, substance use, and mobile use. The internal consistency of this scale was good (Cronbach's alpha $=0.81$ ).

\section{Perceived Severity}

Perceived severity of COVID-19 was recorded with 5 items. Participants rated their perception of how severe the virus was on a five-point Likert scale with the following criteria: Rate of infection, mortality, morbidity, negative impact on social order and negative impact on economic stability (from " 1 = not severe at all" to " 5 = very much severe") (e.g. "How severe do you think the infectiousness of COVID-19 is?", "How severe do you think the morbidity of COVID-19 is?"). Higher scores indicated that the perception of COVID-19 was more severe. The internal consistency of the scale was acceptable (Cronbach's alpha $=0.71)$. 


\section{Perceived Controllability}

Estimation of how the perceived controllability of the COVID-19 situation was measured using 9 items on a five-point Likert scale (from " 1 = totally uncontrollable" to "5 = totally controllable") (e.g. "The ways of transmission", "The infectiousness"). Higher scores reflected participant perceptions that COVID-19 was more controllable. The scale held very good internal consistency (Cronbach's alpha $=0.87$ ).

\section{Perceived Knowledge of COVID-19}

The perception of knowledge about different aspects of COVID-19 (e.g. aetiology, transmission, diagnostic criteria, symptoms etc.) was recorded using 11 items on a five-point Likert scale (from " 1 = totally do not know" to " $5=$ totally know") (e.g. "Its aetiology", "symptoms"). Higher scores implied more knowledge about the difference aspects of COVID-19. The scale also retained very good internal consistency $($ Cronbach's alpha $=0.86)$

\section{Procedure}

Following ethical approval from the Ethical Committee of the Directory of Research and Service Community of the University of Muhammadiyah Malang, the survey was developed in Bahasa Indonesia using Google Forms. The link for the online survey was circulated via a number of avenues using an opportunity sample. The survey could be completed from a personal computer, tablet or smartphone, and it took around 15 minutes to complete with approximately 78 items.

\section{Results}

\section{Descriptive Statistics}

Descriptive statistics for the variables used in this study and their correlations are shown in Table 2.

Table 2. Means, standard deviations, Cronbach's Alpha and correlations between variables

\begin{tabular}{lllrlllllll}
\hline \multicolumn{1}{c}{ Var } & \multicolumn{1}{c}{ X } & SD & \multicolumn{1}{c}{$\boldsymbol{\alpha}$} & \multicolumn{1}{c}{$\mathbf{1}$} & $\mathbf{2}$ & $\mathbf{3}$ & $\mathbf{4}$ & $\mathbf{5}$ & $\mathbf{6}$ & $\mathbf{7}$ \\
\hline 1. IU & 40.25 & 7.75 & 0.83 & - & & & & & \\
2. Know & 39.05 & 6.86 & 0.86 & -0.02 & - & & & & \\
3. Sev & 22 & 2.5 & 0.71 & $0.29^{* * *}$ & $0.16^{* *}$ & - & & & & \\
4. Con & 27.05 & 6.65 & 0.87 & -0.04 & $0.25^{* * *}$ & $-0.18^{* * *}$ & - & & \\
5. EmBe & 53.68 & 10.79 & 0.81 & $0.4^{* * *}$ & -0.07 & $0.24^{* * *}$ & $-0.15^{* *}$ & - & & \\
6. Dep & 6.03 & 4.45 & 0.83 & $0.46^{* * *}$ & $-0.25^{* * *}$ & 0.08 & $-0.12^{*}$ & $0.39^{* * *}$ & - & \\
7. Anx & 6.43 & 4.37 & 0.78 & $0.43^{* *}$ & $-0.19^{* *}$ & $0.13^{* *}$ & -0.08 & $0.41^{* *}$ & $0.68^{* *}$ & - \\
\hline
\end{tabular}

Note: $\mathrm{IU}=$ Intolerance of Uncertainty; Know $=$ Perceived Knowledge; $\mathrm{Sev}=$ Perceived Severity; $\mathrm{Con}=$ Perceived Controllability; $\mathrm{EmBe}=$ Emotional Behavioural Response to COVID-19; Dep = Depression; $\mathrm{Anx}=$ Anxiety. ${ }^{*}=p$ $<.05, * *=p<.01, * * *=p<.001$ 
The results show that all of the variables have acceptable internal consistency with Cronbach's Alpha above 0.70 . Mean scores of each scale revealed that, overall, perceived knowledge about COVID-19 was moderate-high $(\mathrm{M}=39.05$, $\mathrm{SD}=6.86)$, perceived severity was moderate $(\mathrm{M}=22, \mathrm{SD}=2.5)$, perceived control was moderate $(\mathrm{M}=27.05, \mathrm{SD}=6.65)$, emotional and behavioural difficulties were moderate $(\mathrm{M}=53.68, \mathrm{SD}=10.79)$, depression was normal $(\mathrm{M}=6.03, \mathrm{SD}=4.45)$ and anxiety was normal $(\mathrm{M}=6.43, \mathrm{SD}=4.37)$. IU moderately correlated with perceived severity $(r=0.29)$, emotional/behaviour $(r=0.4)$, depression $(r=0.46)$ and anxiety $(r=0.43)$. Although perceived severity weakly correlated with emotional/behaviour $(r=0.24)$ and anxiety $(r=0.13)$, it did not with depression. Subsequently, emotional/behavioural moderately correlated with depression $(r=$ $0.39)$ and anxiety $(r=0.41)$. This correlational matrix revealed that there is no multicollinearity $(r>0.80)$.

\section{Depression Pathway Analysis}

In order to test the first hypothesis we ran a path analysis using AMOS version 23 (Arbuckle, 2014). Direct and indirect effects were estimated using bootstrap analysis. Figure 1 shows the path model and the standardized estimate effects of variables studied.

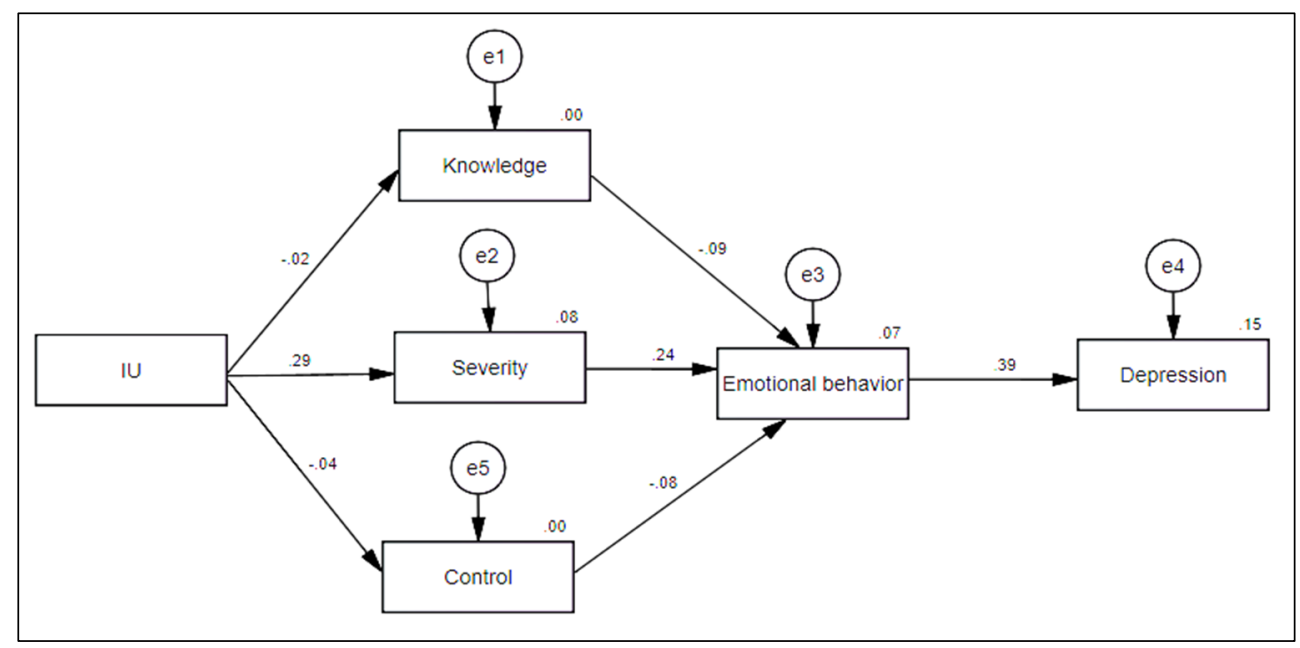

Figure 1. The mediational model of IU to depression via cognitive appraisal variables and emotional/behaviour responses

The direct path from IU to severity was positive and statistically significant (estimated effect $=0.290, p=.01, \mathrm{CI}=0.178,0.283$ ), but not to knowledge or control. On the path from cognitive appraisal variables to emotional and behavioural 
responses, only severity had a positive significant path to emotional/behaviour (estimated effect $=0.242, p=.01, \mathrm{CI}=0.135,0.328$ ). Emotional/behaviour itself forms a significant direct path to depression (estimated effect $=0.387, p=.01, \mathrm{CI}=$ $0.313,0.468$ ).

The indirect path reveals that there is a significant effect of IU to emotional/behaviour via cognitive appraisals (estimated effect $=0.076, p=.10$, CI $=0.030,0.125)$, and also, that there is a significant effect of IU to depression via cognitive appraisals and emotional/behaviour (estimated effect $=0.029, p=.010, \mathrm{CI}$ $=0.010,0.048$ ). Consequently, cognitive appraisals and emotional/behavioural responses mediate the relation between IU and depression. However, since it was only severity that has a significant path from IU, then only severity among the cognitive appraisal variables acts as a mediator. Although perceived severity did not directly correlate with depression, it did contribute in an indirect way by heightening emotional/behaviour. Table 3 shows the indirect effects and their associated $95 \%$ confidence intervals.

Table 3. Direct and indirect effects and $95 \%$ confidence intervals for the depression model

\begin{tabular}{|c|c|c|c|c|c|}
\hline \multirow{2}{*}{ Model pathways } & \multirow{2}{*}{ Estimated effect } & \multirow{2}{*}{ SE } & \multirow{2}{*}{$p$} & \multicolumn{2}{|c|}{$95 \%$ confidence interval } \\
\hline & & & & Lower & Upper \\
\hline \multicolumn{6}{|l|}{ Direct effect } \\
\hline IU $\rightarrow$ Know & -0.025 & 0.052 & 0.616 & -0.133 & 0.076 \\
\hline $\mathrm{IU} \rightarrow \mathrm{Sev}$ & 0.290 & 0.050 & $<0.01$ & 0.178 & 0.283 \\
\hline $\mathrm{IU} \rightarrow \mathrm{Con}$ & -0.040 & 0.060 & 0.422 & -0.161 & 0.100 \\
\hline Know $\rightarrow$ EmBe & -0.087 & 0.048 & 0.068 & -0.179 & 0.017 \\
\hline $\mathrm{Sev} \rightarrow \mathrm{EmBe}$ & 0.242 & 0.052 & $<0.01$ & 0.135 & 0.325 \\
\hline $\mathrm{Con} \rightarrow \mathrm{EmBe}$ & -0.084 & 0.050 & 0.081 & -0.174 & 0.028 \\
\hline $\mathrm{EmBe} \rightarrow \mathrm{Dep}$ & 0.387 & 0.039 & $<0.01$ & 0.313 & 0.468 \\
\hline \multicolumn{6}{|l|}{ Indirect effect } \\
\hline $\mathrm{IU} \rightarrow \mathrm{CA} \rightarrow \mathrm{EmBe}$ & 0.076 & 0.024 & .010 & 0.030 & 0.125 \\
\hline $\mathrm{IU} \rightarrow \mathrm{CA} \rightarrow \mathrm{EmBe} \rightarrow$ Dep & 0.029 & 0.009 & .010 & 0.010 & 0.048 \\
\hline
\end{tabular}

Note: $\mathrm{IU}=$ Intolerance of Uncertainty; Know = Perceived Knowledge; Sev = Perceived Severity; Con = Perceived Controllability; $\mathrm{EmBe}=$ Emotional Behavioural Response to COVID-19; Dep $=$ Depression; $\mathrm{CA}=$ Cognitive appraisal; $\mathrm{SE}=$ standardized error; $p=$ statistical significance; Arrows indicate direction of the pathway; $*=p<$ $.05, * *=p<.01, * * *=p<.001$

\section{Anxiety Pathway Analysis}

We tested the second hypothesis by performing another analysis of the model with anxiety as a dependent variable. Figure 2 demonstrates the path analysis model and standardized estimate effects of variables studied. The pattern of the model is similar with the previous model. 


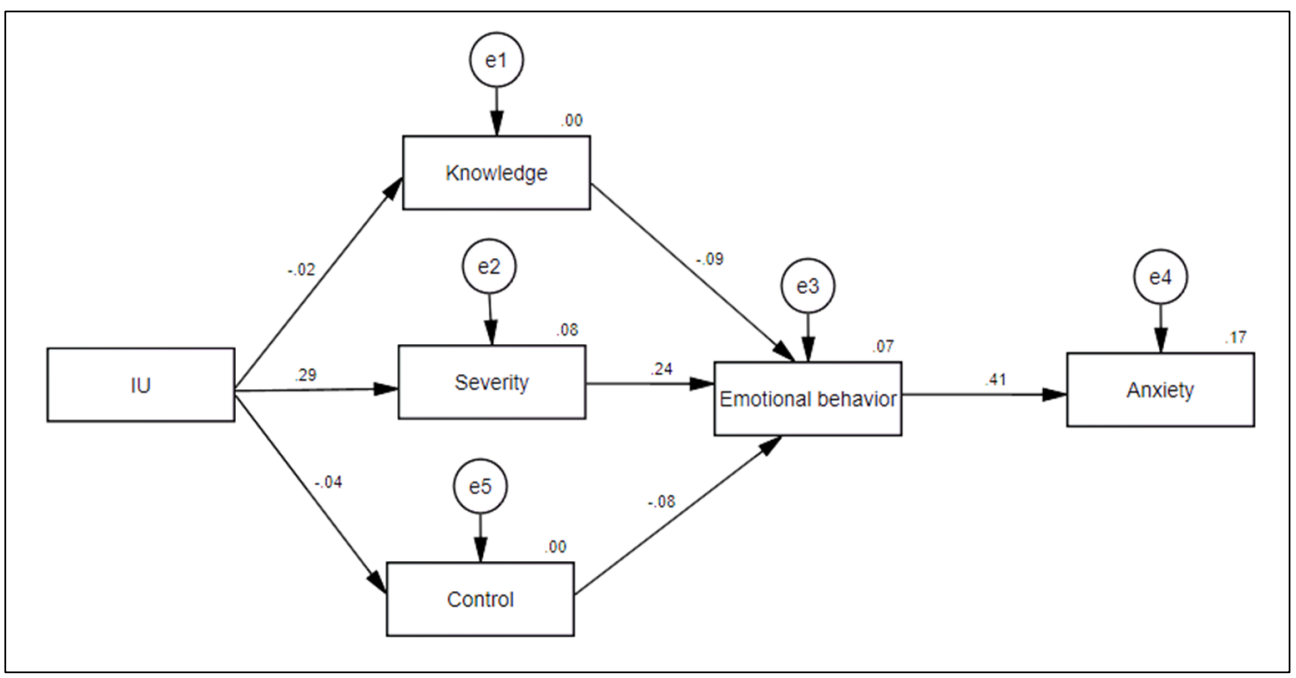

Figure 2. The mediational model of IU to anxiety via cognitive appraisal variables and emotional/behaviour responses

The model remained the same except for a variation in strength in the indirect pathway from IU to anxiety; with a slightly higher estimated effect between $\mathrm{IU}$ and anxiety than with IU and depression (estimated effect $=0.031, p=.01, \mathrm{CI}=$ $0.010,0.055$ ). Table 4 shows the indirect effects and their associated $95 \%$ confidence intervals. Implications are discussed below.

Table 4. Direct and indirect effects and $95 \%$ confidence intervals for the anxiety model

\begin{tabular}{cccccc}
\hline Model pathways & Estimated effect & SE & $\boldsymbol{p}$ & \multicolumn{2}{c}{ 95\% confidence interval } \\
\hline Direct effect & & & & & Lower \\
IU $\rightarrow$ Know & -0.025 & 0.052 & 0.616 & -0.133 & 0.076 \\
IU $\rightarrow$ Sev & 0.290 & 0.050 & $<0.01$ & 0.178 & 0.283 \\
IU $\rightarrow$ Control & -0.040 & 0.060 & 0.422 & -0.161 & 0.100 \\
Know $\rightarrow$ EmBe & -0.087 & 0.048 & 0.068 & -0.179 & 0.017 \\
Sev $\rightarrow$ EmBe & 0.242 & 0.052 & $<0.01$ & 0.135 & 0.325 \\
Con $\rightarrow$ EmBe & -0.084 & 0.050 & 0.081 & -0.174 & 0.028 \\
EmBe $\rightarrow$ Anx & 0.413 & 0.038 & $<0.01$ & 0.337 & 0.498 \\
Indirect effect & & & & & \\
IU $\rightarrow$ CA $\rightarrow$ EmBe & 0.076 & 0.024 & .010 & 0.030 & 0.125 \\
IU $\rightarrow$ CA $\rightarrow$ EmBe $\rightarrow$ Anx & 0.031 & 0.010 & .010 & 0.010 & 0.055 \\
\hline
\end{tabular}

Note: $\mathrm{IU}=$ Intolerance of Uncertainty; Know $=$ Perceived Knowledge; $\mathrm{Sev}=$ Perceived Severity; Con = Perceived Controllability; $\mathrm{EmBe}=$ Emotional Behavioural Response to COVID-19; Anx = Anxiety; CA = Cognitive appraisal; $\mathrm{SE}=$ standardized error; $p=$ statistical significance; Arrows indicate direction of the pathway; $*=p<$ $.05, * *=p<.01, * * *=p<.001$ 


\section{Discussion}

The current study aimed to explore two hypothesized pathways IU and depression and IU and anxiety in an Indonesian sample. Importantly, these pathways were proposed to be serially mediated by cognitive appraisals and emotional and behavioural disturbance during COVID-19. Predictions of indirect and direct association between IU and depression, which the association was serially mediated by cognitive appraisals and emotional and behavioural responses to COVID-19 (i.e., increased negative affect, lower positive affect, anxious symptoms, and sleep disturbance), were supported by the results of the pathway analysis. Similar evidence is also provided for the second pathway with an indirect association between IU and anxiety.

\section{Depression}

In the proposed model for depression, an indirect pathway provides correlational support for the construct's association with IU. Additionally, the other indirect pathway supports the notion that IU increases cognitive risk factors - in this case, by heightening perceived severity - and emotional and behavioural disturbance. This is congruent with previous studies highlighting the role of IU during virus pandemics. For example, IU mediated the relationship between perceived threat of COVID-19, biological rhythms and psychosomatic symptoms (Gica, Kavakli, Durduran, \& Ak, 2020); associated with increased perceptions of threat during the H1N1 crisis, as well as reliance on emotional focused coping, which mediated the relationship between IU and virus-related anxiety (Taha et al, 2014); and outside of a pandemic context, where IU contributed to threat appraisals of women who survived breast cancer (Wonghongkul et al., 2000).

Firstly, it was unsurprising that IU would increase participants' perceived severity of COVID-19. As stated in the introduction, IU is associated with reasoning styles with the tendency to fixate on uncertain aspects of an event and to find this uncertainty threatening. This is supported by neurophysiological alterations in brain regions typically involved with responding to threat, such as the amygdala and anterior insula.

It was, however, surprising that neither perceived controllability nor knowledge about COVID-19 directly associated with IU, or mediated its relationship with emotional and behavioural responses. Possibly, perceived threats are more salient to individuals with higher IU then the aforementioned appraisals because it represents a more direct threat to ones' well-being. Contrarily, perceived knowledge may not be as relevant to IU either because the pandemic remains uncertain regardless of how much information one receives, or because the bias appraisals of IU toward threat renders available knowledge as less important than potential harm. Similarly, in regards to controllability, precautionary actions can reduce uncertainty 
to an extent, but the overarching uncertainty of the pandemic is something that cannot be easily reduced.

Secondly, the direct association between perceived severity and emotional and behavioural disturbance was also expected. Cognitive contributions to threat perception are usually accompanied by an emotional component. In the absence of information, researchers have postulated that risk perception and related behaviour seems to be predicated on people's anticipated emotions (Mellers et al., 1999), gut feelings (Bechara et al., 1997) and past experiences (Wagar \& Dixon, 2006). Theories of a dual-process between cognition and emotion has been proposed, whereby, cognition and emotion contribute to the increased perception of threat (e.g. Van Gelder et al., 2009). Both possibly play a role here although it cannot be confirmed. Likewise, perceived severity has been associated with increased emotional and behavioural disturbance during COVID-19 (Li et al., 2020) and a mediating variable between IU and anxiety alongside emotion-focused coping strategies (opposed to problem-focused) (Taha et al, 2014).

The potential role of IU, therefore, a notion supported by the current findings, is to increase appraisal biases towards threat. Moreover, through a direct association in the correlational matrix and indirectly through the cognitive appraisal mediated pathway; to increase the intensity of adverse emotional and behavioural responses to COVID-19. Appraisals have been positively associated with increased emotional intensity (Ellsworth \& Smith, 1988), as has IU, indicating that affect intensity may be related to the way that a situation is appraised. Although much research has been conducted suggesting that emotions are central to the appraisal process; alternative research emphasizes the role of cognitive appraisals, not just in emotional regulation (Ellsworth \& Scherer, 2003), but also in the production of affective states that are difficult to regulate (Mehu \& Scherer, 2015). It should be noted that cognitive, emotional and behavioural factors are likely mutually reciprocal. It would be fruitful for future research to examine this interplay over time.

As for behavioural responses, many studies have demonstrated that emotional processing in humans modulates motor-related areas (Baumgartner et al., 2007; Hajcak et al., 2007; Oliveri et al., 2003), and that defensive reactions of human beings can be activated intensely in response to aversive stimuli (Mobbs et al., 2007, 2010). As such, when presented with aversive stimuli in a laboratory, it has been shown that the input of aversive stimuli on motor output is a central determinant to the modulation of behaviour by emotion (Junior et al., 2013). These defence cascades may also be part of the regulation process, as emotion increases in response to aversive stimuli, so does the behavioural modulation attempting to reduce it. The increased emotional intensity resulting from the appraisal styles of IU is may engage defence orientated behaviour aimed at coping with the threat of the pandemic. Interestingly, Freeston et al (2020) argued that IU is strongly associated with uncertainty-reducing behaviours designed to avoid or resolve uncertainty distress. It would be interesting to examine the relationship between IU, cognitive appraisals 
and emotion in the context of maladaptive defence cascades in response to a virus pandemic.

Finally, these associations seem to also increase the risk for depression. For the following reasons, this implicates IU as an important risk factor for heightened psychological disturbance and psychopathology during a pandemic like COVID-19, similar to previous studies (e.g. Taha et al., 2014). Indonesians from this sample had, overall, low rates of depression and mild-to-moderate levels of emotional and behavioural responses to COVID-19; but the latter two variables intensified with a higher presence of IU. Thus, although perceived severity did not correlate with depression in the correlational matrix, it did associate with emotional and behavioural difficulties in the pathway, which in turn increased depression. At least in this model, this suggests that variables which increase perceptions of threat, such as IU, are also likely to increase the risk of depression through emotional and behavioural difficulties.

One interpretation of this result is provided by the previously mentioned framework of cognitive risk factors and emotional (and behavioural) dysfunction. Kaiser and Scherer (1998) previously argued for the utility of conceptualizing a variety of clinically salient affective disorders as malfunctions of intraindividual and interindividual regulation of normal emotions. Because powerful emotions require increased efforts of emotional regulation, emotional-related disturbances such as depression are more likely to develop if regulation is impaired (Mathews \& MacLeod, 2005). Moreover, some researchers have suggested that increases in these emotions, possibly due to appraisal biases, present a vulnerability factor for affective disorders (Roseman \& Kaiser, 2001; Scherer \& Brosch, 2009).

This would seem particularly relevant under significant life stress like the current pandemic. Increasing need for intraindividual regulation can deplete psychophysiological resources and opportunities for interindividual regulation are limited. Moreover, pandemics are characterized by a heightening of normal negative affect in response to a threatening event, which would place individuals with cognitive vulnerabilities to affective disorders, such as those with IU, at risk of developing affective disorders. Finally, this could be aggravated by disruptions to daily life due to social restrictions. That is, by increasing the difficultly of maintaining healthy behavioural patterns especially for higher IU individuals who typically engage maladaptive safety seeking behaviours.

\section{Anxiety}

While the main purpose of this article was to demonstrate links between IU and depression in the context of virus pandemics; this paper also provides further evidence of the strong theoretical association between IU and anxiety. Overestimation of threat compared with real threats is a typical feature of anxiety disorders and disorders with an anxious component (Abramowitz \& Blakey, 2020). Accordingly, many models of anxiety are based on threat, such as panic disorder 
(Clark, 1986.), obsessive-compulsive disorder (Salkovskis, 1985) and social anxiety (Clark \& Wells, 1995). As mentioned, this model follows a similar design to Taha et al (2014) study, although the current study demonstrated a link with general anxiety rather than health anxiety.

The current study also supports research during COVID-19 (Tull et al., 2020). IU significantly predicted the health anxiety dimension of bodily vigilance at a one-month follow-up. Furthermore, affective risk assessments and IU uniquely associated with the perceived likelihood that one would develop an illness and that the outcome would be negative at a one month follow up measurement. The role of IU and perceived severity for COVID-19 in the results from this study help to elucidate this relationship.

\section{Uncertainty Distress}

The findings presented here, more so for anxiety than depression, can be contextualized by a recent framework for 'uncertainty distress' (Freeston et al., 2020). This model was developed not only for COVID-19, but also for wider clinical applications to psychological difficulties. Importantly, they highlighted that dispositional IU may be responsible for moderating both perception of threat and uncertainty, which in turn, increases situational uncertainty regarding specific events (e.g. wearing a face mask, social restrictions). Moreover, uncertainty-reducing behaviours can exasperate or prologue both dispositional and situational IU. Actual threat and actual uncertainty are translated through dispositional intolerance into a recursive pattern of perceived threat, perceived uncertainty and situational IU and collectively contributing uncertainty distress.

In both models of the current study, dispositional IU appears to heighten the perceived severity of COVID-19, supporting the notion that individuals with dispositional IU perceive threats higher than people with low IU. The contribution of increased perceived severity on emotional and behavioural difficulties could possibly reflect the uncertainty distress arising from the interplay between perceived threat, perceived uncertainty, situational uncertainty and uncertainty-reducing behaviours as a consequence of dispositional IU. Furthermore, uncertainty distress explains why emotional and behavioural difficulties may lead to anxiety and depression.

One remaining question is how exactly does IU relate to depression theoretically? It's possible that rumination plays a role in mediating between the two consistent with previous studies (i.e., Liao \& Wei, 2011; Yook et al., 2010). Future studies should investigate this link in the context of virus pandemics. Another possible interpretation is that specific emotions generated from a particular appraisal pattern give rise to emotions which associate with an affective disorder (Scherer \& Brosch, 2009). Freeston et al (2020) suggested that although IU is typically characterized by anxiety and worry, it can be accompanied by other emotions. For example, regret, guilt, shame or sadness can be present. If appraisal patterns give rise 
to the latter emotions, it provides a pathway congruent with the notion that depression can arise out of specific maladaptive emotions, such as sadness. Whether this relationship can exist outside the precipitating influence of anxiety remains to be seen. Future studies should also focus on exploring any mediating or moderating effects of anxiety on associations between IU and depression.

\section{Implications for Practice}

The findings of this study have several theoretical and practical implications. Firstly, this study supports the view that IU plays a role in heightening emotional and behavioural disturbance during a pandemic, which may contribute to the development of anxiety and affective disorders like depression. These findings also strengthen the link between IU and perceptions of threat, as well as research highlighting how appraisals are central to the process of regulating ones emotional and behavioural responses during stressful life events like the current pandemic. Accordingly, psychological interventions should consider incorporating IU specific treatment methods to help individuals who are distressed by the uncertainty of COVID-19, placing specific interest on the perception of threat.

It cannot be stated with any certainty, but the results also lend support to arguments that affective disorders arise from maladaptation in normal emotional patterns. This would seem more salient during the pressure of a global pandemic than it would under normal circumstances, given the widespread fear and disruptions to everyday life. This would imply that insofar as possible, helping individuals, especially those with high IU, to manage not only their trait appraisals, but also their emotional states and behaviour during pandemics is important, as it would reduce the risk of developing more severe emotional disturbances. It would be fruitful to profile the relationship between IU and different emotional states to determine whether there are specificities in what emotions give rise to different psychological difficulties. In line with previous research on coping styles, one potential avenue for treatment would be to encourage problem-focused coping styles as opposed to emotion-focused coping styles, which has the potential for reducing distress caused by a pandemic. Future studies should investigate how the promotion of problemfocused coping styles might mitigate the influence of IU during pandemics.

Despite these results, the study does have several limitations. This was a cross-sectional study, so inferences cannot be made of how variables relate overtime, and the correlational nature of the study means that causation cannot be determined between variables. Furthermore, the reliability of the measures was generally acceptable, but should be improved for future studies. Possibly, this reflects the translation process of English language measures into Bahasa Indonesia, and further investigations would benefit from more thorough examination of translated measures before implementation. Also, the study may not be representative of the general population for two reasons. Firstly, the questionnaire was administered online, and so the study did not represent sections of the Indonesian population who 
did not have access to the internet, or that were not familiar with online research platforms. Secondly, the sample was unbalanced, being comprised from more female participants $(72.17 \%)$.

\section{Conclusion}

The global uncertainty surrounding COVID-19 has been compounded for Indonesian citizens by lack of a clear scientific and evidence-based government response. Here, further evidence has been provided that IU is an important risk factor for heightened psychological distress during the current pandemic, as well as potentially increasing the risk for anxious disorders and affective disorders like depression in an Indonesian sample. Particular considerations should be taken surrounding the perceptions of severity toward COVID-19 in higher IU individuals, which appears to be the main cognitive appraisal style which mediates between IU and adverse emotional and behavioural responses. Likewise, direct associations between the emotional and behavioural responses and depression could suggest that depression arises from maladaptation in normal emotions and behaviour. Based on these findings, and those of previous research, educating and helping people who are intolerant to uncertainty to manage the cognitive, emotional and behavioural responses during a pandemic like COVID-19 might be key for reducing adverse psychological outcomes. In the future, greater attention should be paid to the factors that effectively reduce the influence of IU during pandemics, which can inform the design of psychological interventions.

\section{Data Availability}

Data is available by request.

\section{Conflicts of Interest}

The authors of this study can confirm that have no conflicts of interest to disclose.

\section{References}

Abramowitz, J. S., \& Blakey, S. M. (2020). Clinical handbook of fear and anxiety: Maintenance processes and treatment mechanisms. American Psychological Association. 
Almira, T. (2020). Depression and Anxiety in Patients with Corona Virus Disease 2019. Jurnal Penelitian Perawat Profesional, 2(3), 355-360.

Arbuckle, J. L. (2014). Amos (version 23.0)[computer program]. Chicago: IBM SpSS.

Bao, Y., Sun, Y., Meng, S., Shi, J., \& Lu, L. (2020). 2019-nCoV epidemic: Address mental health care to empower society. The Lancet, 395(10224), e37-e38.

Baumgartner, T., Willi, M., \& Jäncke, L. (2007). Modulation of corticospinal activity by strong emotions evoked by pictures and classical music: A transcranial magnetic stimulation study. Neuroreport, 18(3), 261-265.

Bechara, A., Damasio, H., Tranel, D., \& Damasio, A. R. (1997). Deciding advantageously before knowing the advantageous strategy. Science, 275(5304), 1293-1295.

Bigatti, S. M., Steiner, J. L., \& Miller, K. D. (2012). Cognitive appraisals, coping and depressive symptoms in breast cancer patients. Stress and Health, 28(5), $355-361$.

Boelen, P. A., \& Reijntjes, A. (2009). Intolerance of uncertainty and social anxiety. Journal of Anxiety Disorders, 23(1), 130-135.

Boelen, P. A., Vrinssen, I., \& van Tulder, F. (2010). Intolerance of uncertainty in adolescents: Correlations with worry, social anxiety, and depression. The Journal of Nervous and Mental Disease, 198(3), 194-200.

Carleton, R. N. (2012). The intolerance of uncertainty construct in the context of anxiety disorders: Theoretical and practical perspectives. Expert Review of Neurotherapeutics, 12(8), 937-947.

Carleton, R. N. (2016). Into the unknown: A review and synthesis of contemporary models involving uncertainty. Journal of Anxiety Disorders, 39, 30-43.

Carleton, R. N., Norton, M. P. J., \& Asmundson, G. J. (2007). Fearing the unknown: A short version of the Intolerance of Uncertainty Scale. Journal of Anxiety Disorders, 21(1), 105-117.

Clark, D. M. (1986). A cognitive model of panic attacks. In S. Rachman \& J. D. Maser (Eds.). In Panic: Psychological perspectives (pp. 71-89). Lawrence Erlbaum Associates, Inc.

Clark, D. M., \& Wells, A. (1995). A cognitive model of social phobia. In Heimberg, $R$. G., Liebowitz, M. R., Hope, D. A., \& Schneierif, R. (ed), Social Phobia Diagnosis, Assessment and Treatment (p. 69). London, UK: The Guilford Press.

Cox, B. J., Enns, M. W., \& Taylor, S. (2001). The effect of rumination as a mediator of elevated anxiety sensitivity in major depression. Cognitive Therapy and Research, 25(5), 525-534.

de Jong-Meyer, R., Beck, B., \& Riede, K. (2009). Relationships between rumination, worry, intolerance of uncertainty and metacognitive beliefs. Personality and Individual Differences, 46(4), 547-551.

Desclaux, A., Badji, D., Ndione, A. G., \& Sow, K. (2017). Accepted monitoring or endured quarantine? Ebola contacts' perceptions in Senegal. Social Science \& Medicine, 178, 38-45.

DiGiovanni, C., Conley, J., Chiu, D., \& Zaborski, J. (2004). Factors influencing compliance with quarantine in Toronto during the 2003 SARS outbreak. 
Biosecurity and Bioterrorism: Biodefense Strategy, Practice, and Science, 2(4), 265-272.

Dugas, M. J., Buhr, K., \& Ladouceur, R. (2004). The Role of Intolerance of Uncertainty in Etiology and Maintenance.

Dugas, M. J., Marchand, A., \& Ladouceur, R. (2005). Further validation of a cognitive-behavioral model of generalized anxiety disorder: Diagnostic and symptom specificity. Journal of Anxiety Disorders, 19(3), 329-343.

Ellsworth, P. C., \& Scherer, K. R. (2003). Appraisal processes in emotion. Oxford University Press.

Ellsworth, P. C., \& Smith, C. A. (1988). From appraisal to emotion: Differences among unpleasant feelings. Motivation and Emotion, 12(3), 271-302.

Freeston, M. H., Rhéaume, J., Letarte, H., Dugas, M. J., \& Ladouceur, R. (1994). Why do people worry? Personality and Individual Differences, 17(6), 791-802.

Freeston, M. H., Tiplady, A., Mawn, L., Bottesi, G., \& Thwaites, S. (2020). Towards a model of uncertainty distress in the context of Coronavirus (Covid-19).

Gentes, E. L., \& Ruscio, A. M. (2011). A meta-analysis of the relation of intolerance of uncertainty to symptoms of generalized anxiety disorder, major depressive disorder, and obsessive-compulsive disorder. Clinical Psychology Review, 31(6), 923-933.

González-Sanguino, C., Ausín, B., ÁngelCastellanos, M., Saiz, J., López-Gómez, A., Ugidos, C., \& Muñoz, M. (2020). Mental health consequences during the initial stage of the 2020 Coronavirus pandemic (COVID-19) in Spain. Brain, Behavior, and Immunity.

Hajcak, G., Molnar, C., George, M. S., Bolger, K., Koola, J., \& Nahas, Z. (2007). Emotion facilitates action: A transcranial magnetic stimulation study of motor cortex excitability during picture viewing. Psychophysiology, 44(1), 91-97.

Hasanah, U., Fitri, N. L., Supardi, S., \& Livana, P. (2020). Depression Among College Students Due to the COVID-19 Pandemic. Jurnal Keperawatan Jiwa, $8(4), 421-424$.

Jensen, D., Cohen, J. N., Mennin, D. S., Fresco, D. M., \& Heimberg, R. G. (2016). Clarifying the Unique Associations among Intolerance of Uncertainty, Anxiety, and Depression. Cognitive Behaviour Therapy, 45(6), 431-444. https://doi.org/10.1080/16506073.2016.1197308

Joormann, J., \& Gotlib, I. H. (2010). Emotion regulation in depression: Relation to cognitive inhibition. Cognition and Emotion, 24(2), 281-298.

Junior, O. F., Portugal, L. C. L., Alves, R. de C. S., Campagnoli, R. R., Erthal, F. C. S., Mocaiber, I. F., David, I. A., Volchan, E., Oliveira, L., \& Pereira, M. G. (2013). How you perceive threat determines your behavior. Frontiers in Human Neuroscience, 7, 632.

Kaiser, S., \& Scherer, K. R. (1998). Models of 'normal'emotions applied to facial and vocal expression in clinical disorders. J. Flack, f. William \& JD Laird (Eds.), Emotions in Psychopathology: Theory and Research, 81-98.

Khawaja, N., \& McMahon, J. (2011). The relationship of meta-worry and intolerance of uncertainty with pathological worry, anxiety, and depression. Behaviour Change, 28(4), 165-180. 
Koerner, N., \& Dugas, M. J. (2008). An investigation of appraisals in individuals vulnerable to excessive worry: The role of intolerance of uncertainty. Cognitive Therapy and Research, 32(5), 619-638.

Koran Tempo. (2020). Sebagian Warga Jakarta Merasa Kebal Corona-MetroKoran.tempo.co. Koran Tempo. https://koran.tempo.co/read/metro/455028/ sebagian-warga-jakarta-merasa-kebal-corona

Ladouceur, R. (2004). Perceptions among pathological and nonpathological gamblers. Addictive Behaviors, 29(3), 555-565.

Ladouceur, R., Talbot, F., \& Dugas, M. J. (1997). Behavioral expressions of intolerance of uncertainty in worry: Experimental findings. Behavior Modification, 21(3), $355-371$.

Lazarus, R. S. (1966). Psychological stress and the coping process.

Lee-Flynn, S. C., Pomaki, G., DeLongis, A., Biesanz, J. C., \& Puterman, E. (2011). Daily cognitive appraisals, daily affect, and long-term depressive symptoms: The role of self-esteem and self-concept clarity in the stress process. Personality and Social Psychology Bulletin, 37(2), 255-268.

Li, J.-B., Yang, A., Dou, K., Wang, L.-X., Zhang, M.-C., \& Lin, X.-Q. (2020). Chinese public's knowledge, perceived severity, and perceived controllability of the COVID-19 and their associations with emotional and behavioural reactions, social participation, and precautionary behaviour: A national survey.

Liao, K. Y.-H., \& Wei, M. (2011). Intolerance of uncertainty, depression, and anxiety: The moderating and mediating roles of rumination. Journal of Clinical Psychology, 67(12), 1220-1239. https://doi.org/10.1002/jclp.20846.

Lovibond, P. F., \& Lovibond, S. H. (1995). The structure of negative emotional states: Comparison of the Depression Anxiety Stress Scales (DASS) with the Beck Depression and Anxiety Inventories. Behaviour Research and Therapy, 33(3), 335-343.

Mahoney, A. E., \& McEvoy, P. M. (2012). Trait versus situation-specific intolerance of uncertainty in a clinical sample with anxiety and depressive disorders. Cognitive Behaviour Therapy, 41(1), 26-39.

Mathews, A., \& MacLeod, C. (2005). Cognitive vulnerability to emotional disorders. Annu. Rev. Clin. Psychol., 1, 167-195.

McEvoy, P. M., \& Mahoney, A. E. (2011). Achieving certainty about the structure of intolerance of uncertainty in a treatment-seeking sample with anxiety and depression. Journal of Anxiety Disorders, 25(1), 112-122.

Mehu, M., \& Scherer, K. R. (2015). The appraisal bias model of cognitive vulnerability to depression. Emotion Review, 7(3), 272-279.

Mellers, B., Schwartz, A., \& Ritov, I. (1999). Emotion-based choice. Journal of Experimental Psychology: General, 128(3), 332.

Miranda, R., Fontes, M., \& Marroquín, B. (2008). Cognitive content-specificity in future expectancies: Role of hopelessness and intolerance of uncertainty in depression and GAD symptoms. Behaviour Research and Therapy, 46(10), $1151-1159$. 
Mobbs, D., Petrovic, P., Marchant, J. L., Hassabis, D., Weiskopf, N., Seymour, B., Dolan, R. J., \& Frith, C. D. (2007). When fear is near: Threat imminence elicits prefrontal-periaqueductal gray shifts in humans. Science, 317(5841), 1079-1083.

Mobbs, D., Yu, R., Rowe, J. B., Eich, H., FeldmanHall, O., \& Dalgleish, T. (2010). Neural activity associated with monitoring the oscillating threat value of a tarantula. Proceedings of the National Academy of Sciences, 107(47), 2058220586.

Mulya, I. G. N. B. R., Sastrawan, I. G. G., Parastan, R. H., \& Ani, L. S. (2021). Depression, anxiety, and stress levels in Denpasar community during the pandemic of Corona Virus Disease 2019 (COVID-19). Qanun Medika-Medical Journal Faculty of Medicine Muhammadiyah Surabaya, 5(1), 103-111.

Oliveri, M., Babiloni, C., Filippi, M., Caltagirone, C., Babiloni, F., Cicinelli, P., Traversa, R., Palmieri, M., \& Rossini, P. (2003). Influence of the supplementary motor area on primary motor cortex excitability during movements triggered by neutral or emotionally unpleasant visual cues. Experimental Brain Research, 149(2), 214-221.

Parker, C. (2020). Depression in adults: Recognition and management. Evaluation, 14(47), 19.

Paulus, D. J., Talkovsky, A. M., Heggeness, L. F., \& Norton, P. J. (2015). Beyond negative affectivity: A hierarchical model of global and transdiagnostic vulnerabilities for emotional disorders. Cognitive Behaviour Therapy, 44(5), 389-405.

Pompili, M. (2019). Critical appraisal of major depression with suicidal ideation. Annals of General Psychiatry, 18(1), 7.

Prasetyo, Y. T., Castillo, A. M., Salonga, L. J., Sia, J. A., \& Seneta, J. A. (2020). Factors affecting perceived effectiveness of COVID-19 prevention measures among Filipinos during enhanced community quarantine in Luzon, Philippines: Integrating Protection Motivation Theory and extended Theory of Planned Behavior. International Journal of Infectious Diseases, 99, 312-323.

Reuman, L., Jacoby, R. J., Fabricant, L. E., Herring, B., \& Abramowitz, J. S. (2015). Uncertainty as an anxiety cue at high and low levels of threat. Journal of Behavior Therapy and Experimental Psychiatry, 47, 111-119.

Roberts, J., Gilboa, E., \& Gotlib, I. (1998). Ruminative response style and vulnerability to depressive episodes: Factor components, mediating processes, and episode duration. Cognitive Therapy and Research, 22, 401-425.

Roseman, I., \& Kaiser, S. (2001). Applications of appraisal theory to understanding, diagnosis, and treating emotional pathology. Appraisal Processes in Emotion: Theory, Methods, Research, 249-267.

Rosser, B. A. (2019). Intolerance of uncertainty as a transdiagnostic mechanism of psychological difficulties: A systematic review of evidence pertaining to causality and temporal precedence. Cognitive Therapy and Research, 43(2), 438-463.

Salkovskis, P. M. (1985). Obsessional-compulsive problems: A cognitive-behavioural analysis. Behaviour Research and Therapy, 23(5), 571-583. 
Scherer, K. R. (1984). On the nature and function of emotion: A component process approach. Approaches to Emotion, 2293(317), 31.

Scherer, K. R., \& Brosch, T. (2009). Culture-specific appraisal biases contribute to emotion dispositions. European Journal of Personality: Published for the European Association of Personality Psychology, 23(3), 265-288.

Siste, K., Hanafi, E., Lee Thung Sen, H. C., Adrian, L. P. S., Limawan, A. P., Murtani, B. J., \& Suwartono, C. (2020). The Impact of Physical Distancing and Associated Factors Towards Internet Addiction Among Adults in Indonesia During COVID-19 Pandemic: A Nationwide Web-Based Study. Frontiers in Psychiatry, 11.

Spasojević, J., \& Alloy, L. B. (2001). Rumination as a common mechanism relating depressive risk factors to depression. Emotion, 1(1), 25.

Sunjaya, D. K., Herawati, D. M. D., \& Siregar, A. Y. (2021). Depressive, anxiety, and burnout symptoms on health care personnel at a month after COVID-19 outbreak in Indonesia. BMC Public Health, 21(1), 1-8.

Suryahadi, A., Al Izzati, R., \& Suryadarma, D. (2020). Estimating the impact of covid-19 on poverty in Indonesia. Bulletin of Indonesian Economic Studies, $56(2), 175-192$.

Taha, S., Matheson, K., Cronin, T., \& Anisman, H. (2014). Intolerance of uncertainty, appraisals, coping, and anxiety: The case of the 2009 H 1 N 1 pandemic. British Journal of Health Psychology, 19(3), 592-605.

Tanovic, E., Gee, D. G., \& Joormann, J. (2018). Intolerance of uncertainty: Neural and psychophysiological correlates of the perception of uncertainty as threatening. Clinical Psychology Review, 60, 87-99.

Tull, M. T., Barbano, A. C., Scamaldo, K. M., Richmond, J. R., Edmonds, K. A., Rose, J. P., \& Gratz, K. L. (2020). The prospective influence of COVID-19 affective risk assessments and intolerance of uncertainty on later dimensions of health anxiety. Journal of Anxiety Disorders, 75, 102290.

Van Gelder, J., De Vries, R. E., \& Van Der Pligt, J. (2009). Evaluating a dual-process model of risk: Affect and cognition as determinants of risky choice. Journal of Behavioral Decision Making, 22(1), 45-61.

Wagar, B. M., \& Dixon, M. (2006). Affective guidance in the Iowa gambling task. Cognitive, Affective, \& Behavioral Neuroscience, 6(4), 277-290.

Wang, C., Pan, R., Wan, X., Tan, Y., Xu, L., McIntyre, R. S., Choo, F. N., Tran, B., Ho, R., \& Sharma, V. K. (2020). A longitudinal study on the mental health of general population during the COVID-19 epidemic in China. Brain, Behavior, and Immunity.

Wonghongkul, T., Moore, S. M., Musil, C., Schneider, S., \& Deimling, G. (2000). The influence of uncertainty in illness, stress appraisal, and hope on coping in survivors of breast cancer. Cancer Nursing, 23(6), 422-429.

Xin, M., Luo, S., She, R., Yu, Y., Li, L., Wang, S., Ma, L., Tao, F., Zhang, J., \& Zhao, J. (2020). Negative cognitive and psychological correlates of mandatory quarantine during the initial COVID-19 outbreak in China. American Psychologist, 75(5), 607. 
Y1ldırım, M., \& Güler, A. (2020). COVID-19 severity, self-efficacy, knowledge, preventive behaviors, and mental health in Turkey. Death Studies, 1-8.

Yook, K., Kim, K.-H., Suh, S. Y., \& Lee, K. S. (2010). Intolerance of uncertainty, worry, and rumination in major depressive disorder and generalized anxiety disorder. Journal of Anxiety Disorders, 24(6), 623-628.

Yuniardi, M. S. (2020). Should we shoot intolerance of uncertainty for anxiety disorders? Data from the UK and Indonesia. International Journal of Psychosocial Rehabilitation, 24(08), 5134-5146.

Zhong, Y., Liu, W., Lee, T.-Y., Zhao, H., \& Ji, J. (2020). Risk Perception, Knowledge, Information Sources and Emotional States among COVID-19 Patients in Wuhan, China: A Cross-sectional Survey. Nursing Outlook. 